TITLE:

\title{
Three topologically nontrivial choreographic motions of three bodies
}

\author{
AUTHOR(S): \\ uvakov, Milovan; Shibayama, Mitsuru
}

\section{CITATION:}

uvakov, Milovan ...[et al]. Three topologically nontrivial choreographic motions of three bodies. Celestial Mechanics and Dynamical Astronomy 2016, 124(2): 155-162

ISSUE DATE:

2016-02

URL:

http://hdl.handle.net/2433/240755

\section{RIGHT:}

This is a post-peer-review, pre-copyedit version of an article published in Celestial Mechanics and Dynamical Astronomy. The final authenticated version is available online at: http://dx.doi.org/10.1007/s10569-015-9657-9.; This is not the published version. Please cite only the published version.; この論文は出版社版でありません。引用の際には出 版社版をご確認ざ利用ください。 
Noname manuscript No.

(will be inserted by the editor)

\title{
Three Topologicaly Nontrivial Choreographic Motions of Three Bodies
}

\author{
Milovan Šuvakov • Mitsuru Shibayama
}

Received: date / Accepted: date

\begin{abstract}
Choreographies are periodic orbits in which all bodies move on the same trajectory with equal time delay. The best known three-body choreography is figure-eight orbit. Here we introduce a search method specialised for choreographies and present three new orbits with vanishing angular momentum that are the first clear examples of choreographies that cannot be described as $k$-th powers of the figure-eight solution, according to the topological classification of orbits. We have also found seventeen new "powers of the eight" choreographies. According to our numerical computation, one of two distinct $k=7$ choreographies is linearly stable.
\end{abstract}

Keywords Three-body problem · Choreography · Stability

\section{Search of periodic solutions}

The first stable three-body choreographic orbit without angular momentum was found by Moore in 1993 [1]. A formal variational existence proof for such a solution was given by Chenciner and Montgomery [2]. A large number (345) of three-body choreographies with non-zero angular momentum were found by Simó [3], but they are all highly unstable [4], and of undetermined topological type. In Refs. $[3,5,6]$, Simó and co-authors showed several orbits including

\section{M. Šuvakov}

Institute of Physics, University of Belgrade, Pregrevica 118, Zemun-Beograd 11080, Serbia. On leave of absence, presently at Serbian Ministry of Education, Science and Technological Development, Nemanjina 22, 11050 Beograd, Serbia

E-mail: suki@ipb.ac.rs

M. Shibayama

Division of Mathematical Science, Graduate School of Engineering Science, Osaka University; 1-3 Machikaneyama-cho, Toyonaka, Osaka 560-8531, present address: Department of Applied Mathematics and Physics, Graduate School of Informatics, Kyoto University, Yoshida-honmachi, Sakyo-ku, Kyoto 606-8501, Japan

E-mail: shibayama@sigmath.es.osaka-u.ac.jp, shibayama@amp.i.kyoto-u.ac.jp 


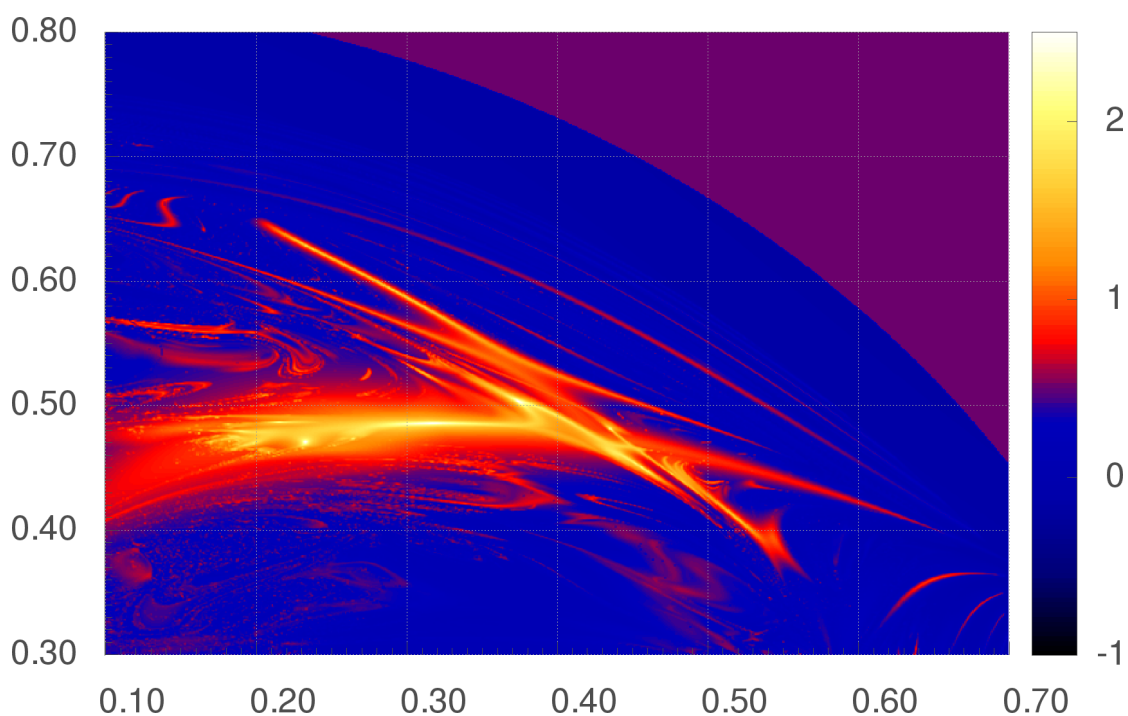

Fig. 1 The decimal logarithm of the reciprocal of the cyclic permutation proximity function $-\log _{10} d_{\hat{P}}\left(\mathbf{X}_{\mathbf{0}}, T_{0}\right)$ in the search window. On $\mathrm{x}$-axis are the values of the initial velocity $\dot{x}_{1}(0) \in(0.1,0.7)$, and on the y-axis are the values of the initial velocity $\dot{y}_{1}(0) \in(0.3,0.8)$. The bright dots represent close to zero values of $d_{\hat{P}}\left(\mathbf{X}_{0}, T_{0}\right)$.

one choreography that they called "satellites of the eight". Ref. [6] is quite clear about their stability: "All the choreographies found, except the eight, are unstable". Unfortunately, in the meantime the initial conditions of these solutions have been lost.

Galán et al. [7] have numerically studied the stability and bifurcations of the figure-eight orbits with almost equal masses. Kapela and Simó [8] have provided a computer assisted proof of the existence (and of linear stability), of the figure-eight orbit, and later they [9] also gave a computer assisted proof of the Kolomogorov-Arnold-Moser (KAM) stability. Thus it follows from the Poincaré-Birkhoff theorem, that there are infinitely many periodic solutions near the figure-eight. Therefore, we expect that at least some of these solutions would also be "simple" choreographies, i.e., satellites of the eight. There was no evidence, however, prior to present work, that there are choreographies with vanishing angular momentum that are not satellites of the figure-eight. Here we present, for the first time, three examples of such orbits.

We identify curves that can be modified continuously one into another in the shape space without collisions. We call their equivalence classes "homotopy classes". We can classify periodic orbits according to their homotopy classes. For example, a power- $k$ figure-eight solution has the same homotopy class as a curve that repeats the figure-eight solution $k$ times within one period. We shall call a "slalom" any solution with the same homotopy class as the power $k$ figure-eight solution. 
In a recent paper [11] one of us (M. Š.) presented the results of a search in the vicinity of the figure-eight orbit. He found eleven solutions that can be described as some "power of the figure-eight solution" in the sense of the topological classification method. Such solutions were called "slaloms" because of their appearance on the shape sphere. One of these solutions, with the seventh power of the figure-eight, is a choreography; some numerical evidence of its stability was shown, but not a definitive proof. Here we present the results of a calculation of the stability coefficients that prove the linear stability of this orbit.

In this paper we specifically searched for choreographies with vanishing angular momentum by modifying the scanning method used in Refs. [10-12]. Insted of minimising return proximity function we used cyclic permutation proximity function defined as:

$$
d_{\hat{P}}\left(\mathbf{X}_{\mathbf{0}}, T_{0}\right)=\min _{t \leq T_{0}}\left|\hat{P} \mathbf{X}(t)-\mathbf{X}_{\mathbf{0}}\right|,
$$

where $\mathbf{X}(t)=\left(\mathbf{r}_{\mathbf{1}}(t), \mathbf{r}_{\mathbf{2}}(t), \mathbf{r}_{\mathbf{3}}(t), \mathbf{p}_{\mathbf{1}}(t), \mathbf{p}_{\mathbf{2}}(t), \mathbf{p}_{\mathbf{3}}(t)\right)$ is a 12 -vector in the phase space (all three bodies' Cartesian coordinates and velocities), and $\mathbf{X}_{\mathbf{0}}=\mathbf{X}(0)$ is 12 -vector describing the initial condition, and $\hat{P}$ is a cyclic permutation of the body indeces in the phase space vector. Whereas the return proximity function is zero for any periodic solution, cyclic permutation proximity function is zero only when solution is choreography.

We found a number of new choreographies, three of which are not satellites of the figure-eight, but of new hitherto unknown topological types. The remaining 17 choreographies are of the (familiar) "satellite-of-eight" type. We also show that one of two distinct $k=7$ choreographies is linearly stable, only the second stable choreography, after the figure-eight.

We only consider orbits with zero angular momentum here, because: 1) the original figure-eight solution also has zero angular momentum; and 2) we searched in the same two-dimensional sub-space of the full phase space of initial conditions in which other satellites of the figure-eight orbit were found previously, see Refs. [10-12]. That subspace corresponds to orbits with zero angular momenta. Of course, one can also search for orbits with non-vanishing angular momenta, but that would introduce a third parameter (dimension of the search sub-space), which would make the search less efficient. It is more efficient to first find periodic orbits with zero angular momentum and then to continue them to non-vanishing values. We have used the shape-sphere variables, Refs. $[10,12]$, in order to reduce the system to three degrees of freedom and thus simplify the computation of the (linear) stability coefficients.

We focussed our numerical search on a square search window with unit sides in the initial velocities plane. The equations of motion were integrated up to time $T_{0}=\frac{1}{3} T=66$ for each initial condition out of $1000 \times 1000$ possibilities (points on the grid) within the search window. The cyclic permutation proximity function $d_{\hat{P}}\left(\mathbf{X}_{\mathbf{0}}, T_{0}\right)$ was calculated and the negative logarithm of 


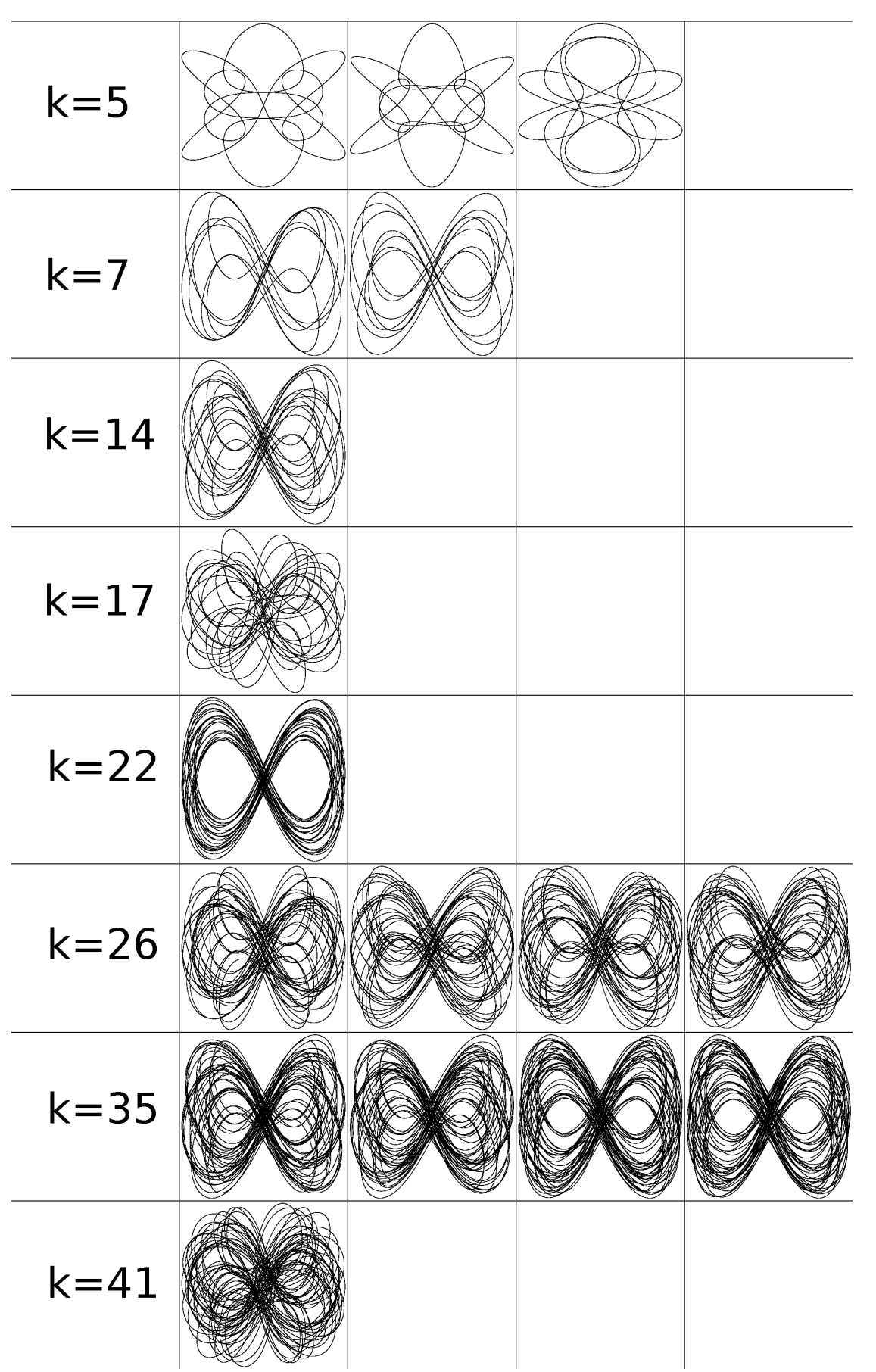

Fig. 2 New choreographies in real space devided in rows by slalom power $k$ asociated with topology (i.e. $a b A B^{k}$ is homotopy class of the orbit). The axes are the original axes in physical space. 
Table 1 The initial conditions (velocities) and the "best pair value" ( We have computed them by using three kinds of argorithm, chosen the closest two values, and called the mean of the two value "best pair value".) of the period of three-body choreographies. $\dot{x}_{1}(0), \dot{y}_{1}(0)$ are the first particle's initial velocities in the $\mathrm{x}$ - and y-directions, respectively, $T$ is the period, $k$ is slalom power (i.e. $(a b A B)^{k}$ is the homotopy class of the orbit, except for orbits \# 22-24, which are not slaloms; for their homotopy classes, see Table 2). The first row, which corresponds to the figure-eight choreography, is shown for comparison.

\begin{tabular}{lcccc}
\hline \hline No. & $\dot{x}_{1}(0)$ & $\dot{y}_{1}(0)$ & $\mathrm{T}$ & $k$ \\
\hline \hline 1 & 0.347116899108889 & 0.532724945068359 & 6.32591 & 1 \\
\hline 2 & 0.322184765624991 & 0.647989160156249 & 51.3958 & 5 \\
3 & 0.257841699218752 & 0.687880761718747 & 55.6431 & 5 \\
4 & 0.568991007042164 & 0.449428951346711 & 51.9645 & 5 \\
5 & 0.500617577931285 & 0.493599179268241 & 63.0165 & 7 \\
6 & 0.209661500479354 & 0.52570238916291 & 33.8615 & 7 \\
7 & 0.255430947113037 & 0.516385839653015 & 35.0431 & 7 \\
8 & 0.410354983519788 & 0.551985420227794 & 57.5453 & 7 \\
9 & 0.477098825943472 & 0.527902384400369 & 133.053 & 14 \\
10 & 0.130360568892222 & 0.526450460061792 & 76.2293 & 17 \\
11 & 0.272790828323364 & 0.532396825027466 & 119.293 & 22 \\
12 & 0.190923947143555 & 0.52171879918566 & 121.551 & 26 \\
13 & 0.221436750501395 & 0.508906476187706 & 121.343 & 26 \\
14 & 0.340328994750794 & 0.578358639621538 & 194.947 & 26 \\
15 & 0.200740359497072 & 0.532146307373045 & 126.929 & 26 \\
16 & 0.273620831298869 & 0.513234869384665 & 132.665 & 26 \\
17 & 0.217541669535648 & 0.514854808068074 & 165.455 & 35 \\
18 & 0.202907533335709 & 0.521482286801917 & 165.797 & 35 \\
19 & 0.234080800616742 & 0.53387583102584 & 179.459 & 35 \\
20 & 0.289649049395323 & 0.518680000030657 & 186.853 & 35 \\
21 & 0.178043164539338 & 0.524061330985931 & 190.371 & 41 \\
22 & 0.698073236083981 & 0.328500769042967 & 100.846 & $*$ \\
23 & 0.108978254696727 & 0.547796320721508 & 89.3028 & $*$ \\
24 & 0.226986094451136 & 0.616036339068786 & 152.556 & $*$ \\
\hline
\end{tabular}

the function is shown in Fig. 1. For each local minimum of the cyclic return proximity function lower then $10^{-2}$ (corresponding to the bright dots in Fig. 1) on this grid we used a simple gradient descent algorithm to find the position of the minimum (root) more accurately. All of the minima with cyclic permutation proximity function below $10^{-6}$ are listed in Table 1 .

The initial condition for the figure-eight choreography is labeled by 1 in Table 1. The initial configuration for all these orbits is the Euler one (collinear, equidistant), see Refs. [10-12]. All other displayed orbits in Table 1 are slaloms, i.e., figure-eight orbit satellites to some (integer) power $k$, except for the last three orbits (labeled 22-24), which correspond to new types of choreographies with different, more complicated topologies than the satellite of the eight (see Table 2).

There are three singular points on the shape sphere. The symbols $a$ and $b$ stand for the homotopy class of curves winding around one and another point once, respectively. $A$ and $B$ stand for the curve of $a$ and $b$ in opposite direction, respectively. The class of curves winding the other singularity can 


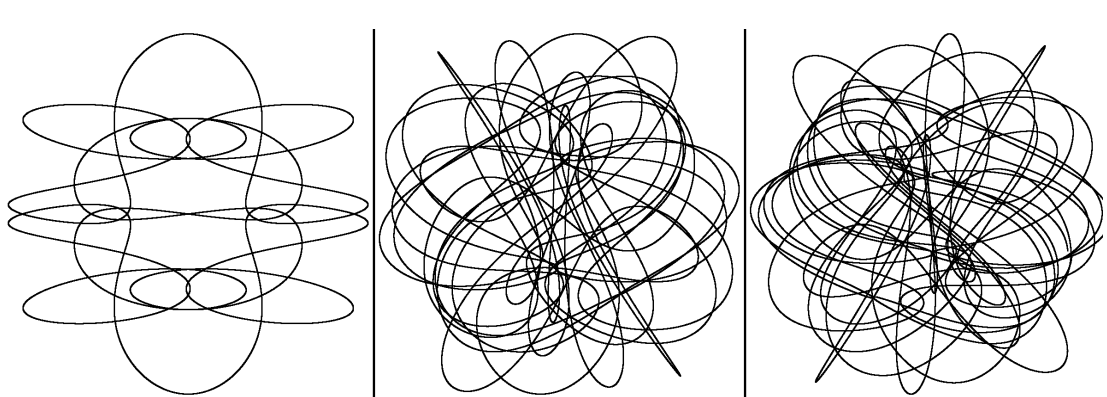

Fig. 3 Three non-slalomic orbits in real space (denoted by 22, 23, and 24 in Table 1, for their homotopy classes, see Table 2).

Table 2 Free-group elements for three non-slalomic choreographic orbits \# 22-24

\begin{tabular}{|c|c|}
\hline No. & Free-group element \\
\hline 222 & 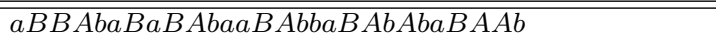 \\
\hline 23 & $\begin{array}{l}a B A b a B B a b A B a b A B a b A B a B A b a B A b a B A b a b A B a b A \\
B a b A B a b a B A b a B A b a B A b A B a b A B a b A B a b A A b a B A b\end{array}$ \\
\hline 24 & $\begin{array}{l}a B A b a B A b b A B a b A B a b A B a b A b a B A b a B A b a B A b a B A \\
B a b A B a b A B a b A B A b a B A b a B A b a B A b a B a b A B a b A B a \\
b A B a a B A b a B A b\end{array}$ \\
\hline
\end{tabular}

be represented by $a b$. The set of words has the group structure and is called free-group. The free-group elements describing the topologies of these three non-slalomic choreographies are listed in Table 2. The real-space trajectories of these three choreographies can be seen in figure 3 .

In order to illustrate the complexity and the symmetry of these solutions, we have plotted the trajectory of the simplest one (22) on the shape sphere, Fig. 4. Note the symmetry of this orbit under rotations through $2 \pi / 3$ about the z-axis, which is the tell-tale sign of a choreography, and the small triangleshaped "empty space" near the North and South Poles in the right-hand-side panel of Fig. 4. The other two choreographies $(\#=23,24)$ are (much) more complicated than this one, so that their plots on the shape sphere would not be very illuminating, though they share the above rotation symmetry and the triangular "exclusion zone" near the Poles.

\section{Stability}

We transformed the original differential equations in Cartesian coordinates to ones in the shape space $[10,12]$. The corresponding phase space is sixdimensional. The equations still form a Hamiltonian system, and hence conserve the energy. We calculated the variational equation along each of the obtained choreographic solutions. The monodromy matrix is symplectic. The eigenvalues consist of three pairs $\left(\lambda_{i}, \lambda_{i}^{-1}\right)(i=1,2,3)$. Because of energy conservation, one of the eigenvalues is unity, $\left(\lambda_{1}:=1\right)$, and then the conjugate 
Table 3 Periods $\mathrm{T}$ of three-body orbits rescaled to a common energy $E=-1 / 2$, together with the rescaling factor $\lambda=-2 E$. $\mathrm{T}_{\# 1}$ is the period of figure-eight orbit and $k$ is the slalom power (i.e. $(a b A B)^{k}$ is homotopy class of the orbit). Remark that No. 7 and 8 are the same solution, but have different energy before rescaling.

\begin{tabular}{lcccc}
\hline \hline No. & $\lambda$ & $\mathrm{T}$ & $\mathrm{T} / \mathrm{T}_{\# 1}$ & $k$ \\
\hline \hline 1 & 2.57428 & 26.1281 & 1 & 1 \\
\hline 2 & 1.85784 & 130.149 & 4.98118 & 5 \\
3 & 1.76203 & 130.146 & 4.98106 & 5 \\
4 & 1.84558 & 130.288 & 4.98652 & 5 \\
5 & 2.03445 & 182.863 & 6.99870 & 7 \\
6 & 3.07807 & 182.863 & 6.99870 & 7 \\
7 & 3.00860 & 182.873 & 6.99910 & 7 \\
8 & 2.16153 & 182.873 & 6.99910 & 7 \\
9 & 1.96217 & 365.705 & 13.9966 & 14 \\
10 & 3.23514 & 443.569 & 16.9767 & 17 \\
11 & 2.85283 & 574.815 & 21.9999 & 22 \\
12 & 3.14815 & 678.954 & 25.9856 & 26 \\
13 & 3.15188 & 678.998 & 25.9873 & 26 \\
14 & 2.29806 & 679.140 & 25.9927 & 26 \\
15 & 3.05914 & 679.144 & 25.9929 & 26 \\
16 & 2.97033 & 679.145 & 25.9929 & 26 \\
17 & 3.12560 & 914.284 & 34.9923 & 35 \\
18 & 3.12131 & 914.285 & 34.9924 & 35 \\
19 & 2.96110 & 914.415 & 34.9974 & 35 \\
20 & 2.88245 & 914.415 & 34.9974 & 35 \\
21 & 3.16196 & 1070.370 & 40.9664 & 41 \\
\hline
\end{tabular}

eigenvalue $\lambda_{1}^{-1}$ is also unity. Consequently, there are two pairs of non-trivial (non-unity) eigenvalues. Only the solution with initial condition labels \#7 and \#8 in Tables 1 and 3 (which correspond to the same solution) is linearly stable. The characteristic polynomial is

$$
\lambda^{4}+a_{1} \lambda^{3}+a_{2} \lambda^{2}+a_{1} \lambda+1=0,
$$

with

$$
a_{1}=-0.8095, \quad a_{2}=1.698 .
$$

Its four roots are the eigenvalues $\lambda_{i}$ of the monodromy matrix:

$$
\lambda_{2}^{ \pm}=-0.1388 \pm 0.9903 i, \quad \lambda_{3}^{ \pm}=0.5435 \pm 0.8394 i .
$$

have moduli equal to unity, within their margins of error: $\left|\lambda_{2}^{ \pm}\right|=0.99998$ and $\left|\lambda_{3}^{ \pm}\right|=0.999992$, which means that the system is linearly stable.

As proposed in Ref. [13], the period $T$ of a slalom three-body orbit that is the $k$-th satellite of the figure- 8 choreography and is rescaled to a common energy, say to $E=-1 / 2$, ought to stand in the ratio to the figure- 8 period $T_{\# 1}$ as the integer $k=1,2,3, \ldots$ that characterizes the topology of the orbit on the shape sphere: $k=T / T_{\# 1}$. This proposition is checked in Table 3 for the new orbits \# 2 - 21 reported here. It should be clear that the slalom orbits follow the proposed law. 

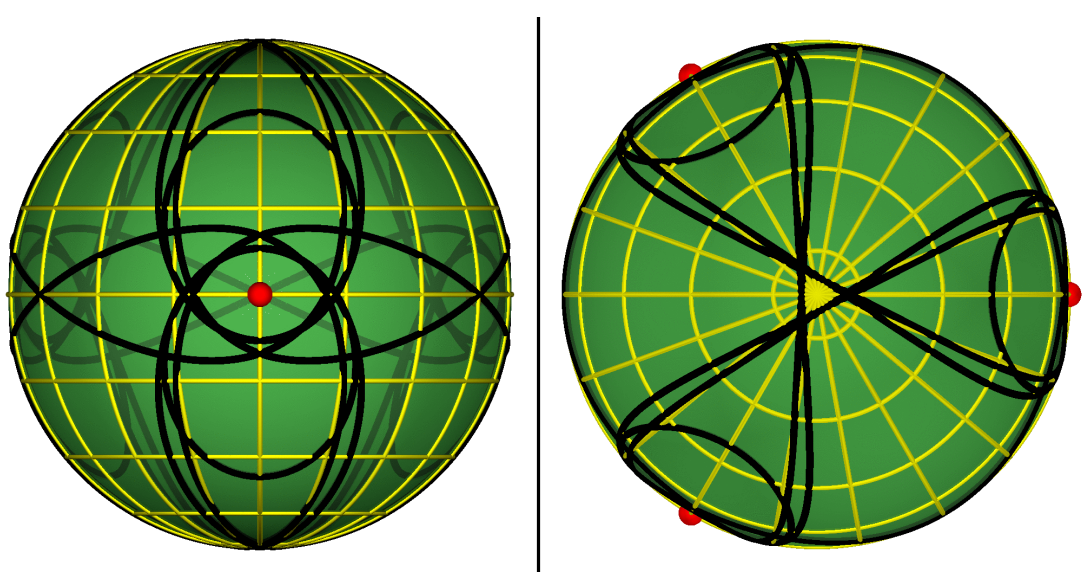

Fig. 4 Shape sphere projection of the simplest non-slalomic choreography (denoted by 22 in Table 1). The curves are in the shape sphere in three dimensional (xyz-)space. This was used by Chenciner-Montgomery's proof. The figures show the projection to $x y$-plane.

\section{Conclusion}

As the result of our new method for direct searching for choreographic solutions to the three-body problem, we have presented 20 new distinct choreographies. Three solutions are the first zero-angular-momentum choreographies found that are not satellites of the eight. The remaining 17 solutions can be described as slaloms with powers $k=5,7,14,17,22,26,35,41$; they satisfy the integerlaw for periods at common energy, as suggested in Ref. [13]. One of two slalom choreographies with $k=7$ (with i.c. labeled as 7 and 8 in Table 1), is linearly stable, only the second stable choreography, after (the famous) Moore's "figureeight" solution.

\section{Acknowledgement}

We would like to thank V. Dmitrašinović for his help in the initial and the final stages of this work, and to Dr. Ayumu Sugita (Osaka City University) for alerting him to the fourth-significant-digit error in the period of Moore's figure8 orbit published in Table II of Ref. [12], as well as for lending us his program for the evaluation of periods. This work was supported by the Serbian Ministry of Education, Science and Technological Development under grant numbers OI 171037 and III 41011, and the Japan Society for the Promotion of Science (JSPS), Grant-in-Aid for Young Scientists (B) No. 26800059. The computing cluster Zefram (zefram.ipb.ac.rs) at the Institute of Physics Belgrade has been extensively used for calculations. 


\section{References}

1. Moore, C.: Braids in classical gravity, Phys. Rev. Lett. 70, 3675-3679 (1993).

2. Chenciner, A., Montgomery, R.: A Remarkable Periodic Solution of the Three-Body Problem in the Case of Equal Masses, Annals of Mathematics, 152, 881-901 (2000).

3. Simó, C.: Dynamical properties of the figure eight solution of the three-body problem, Contemp. Math. 292, 209-228 (2002).

4. Simó, C.: (private communication, 2012).

5. Chenciner, A., Gerver, J., Montgomery, R., Simó, C.: Simple Choreographic Motions of N Bodies: A Preliminary Study, pp. 287-308 in Geometry, Mechanics, and Dynamics, Springer (2002),

6. Simó, C.: New families of solutions in N-body problems, pp. 101-115 in European Congress of Mathematics, Vol. I (Barcelona, 2000), Progress in Mathematics, 201, Birkhäuser, Basel, (2001).

7. Galán, J., Muñoz-Almaraz, F. J., Freire, E., Doedel, E., Vanderbauwhede, A.: Stability and Bifurcations of the Figure- 8 Solution of the Three-Body Problem, Phys. Rev. Lett. 88, 241101 (2002).

8. Kapela T., Simó,C.: Computer assisted proofs for nonsymmetric planar choreographies and for stability of the Eight, Nonlinearity 20, 1241-1255 (2007).

9. Kapela T., Simó, C.: Rigorous KAM results around arbitrary periodic orbits for Hamiltonian Systems, preprint, arXiv:1105.3235 (2011).

10. Šuvakov M., Dmitrašinović, V.: Three Classes of Newtonian Three-Body Planar Periodic Orbits, Phys. Rev. Lett. 110 (11), 114301 (2013)

11. Šuvakov, M.: Numerical Search for Periodic Solutions in the Vicinity of the Figure-Eight Orbit: Slaloming around Singularities on the Shape Sphere Celest. Mech. Dyn. Astron. 119, 369-377 (2014).

12. Šuvakov M., Dmitrašinović, V.: A guide to hunting periodic three-body orbits, Am. J. Phys. 82, 609-619 (2014).

13. Dmitrašinović, V., Šuvakov, M.: Topological Dependence of Kepler's Third Law for Collisionless Periodic Three-Body Orbits with Vanishing Angular Momentum and Equal Masses, Phys. Lett. A 379, 1939-1945 (2015). 